Ann. Biol. anim. Bioch. Biophys., I963, 3 (4), 405-425

\title{
RÉINNERVATION D'UN MUSCLE STRIÉ PAR DES FIBRES PRÉGANGLIONNAIRES PARASYMPATHIQUES. APPLICATION A L'ENREGISTREMENT DE L'ACTIVITÉ DES FIBRES EFFÉRENTES VAGALES CHEZ L'ANIMAL ÉVEILLÉ (1)
}

\author{
M. DUSSARDIER ( $\left.{ }^{2}\right)$ \\ avec la collaboration technique de Colette Lavenet et de J. L. Durel \\ Station centrale de Physiologie animale, \\ Centre national de Recherches zootechniques, Jouy-en-Josas, (Seine-et-Oise)
}

SOMMAIRE

Crâce à une suture nerveuse croisée unilatérale vago-phrénique, faite en arrière du lieu d'émergence du nerf récurrent, le diaphragme a été réinnervé par des fibres motrices vagales chez 6 moutons et 6 boucs. Une fenêtre réalisée dans la paroi thoracique a permis de transformer le diaphragme en muscle sous-cutané, de telle sorte que son activité électromyographique puisse être facilement captée chez l'animal éveillé grâce à des aiguilles de Bronk. Les enregistrements ainsi obtenus et les contrôles histologiques effectués après abattage des animaux ont permis d'établir les faits suivants :

I) des fibres vagales ont envahi le phrénique et atteint le diaphragme;

2) elles ont constitué des plaques motrices sur ce muscle ;

3) ces plaques motrices sont fonctionnelles;

4) l'activité des fibres diaphragmatiques réinnervées par le vague gauche est synchrone de la contraction gastrique provoquée par le vague droit resté en place.

Ces divers faits montrent que les fibres vagales préganglionnaires normalement destinées à l'estomac peuvent constituer des jonctions neuromusculaires fonctionnelles sur des fibres musculaires striées.

L'activité électrique des unités motrices réinnervées peut être recueillie facilement chez l'animal éveillé. Les sutures nerveuses croisées hétérogènes peuvent donc constituer une technique d'étude de l'activité unitaire des fibres efférentes vagales chez l'animal chronique. I960).

(1) Ces recherches constituent une partie de la thèse de Doctorat ès-Sciences de l'auteur (Dussardier,

${ }^{(2)}$ Adresse actuelle : Laboratoire de Physiologie générale, Faculté des Sciences, place Victor-Hugo, Marseille III ${ }^{e}$, France. 


\section{INTRODUCTION}

Flourens ayant montré dès I 828 la possibilité de réaliser des sutures nerveuses, les physiologistes se préoccupèrent ensuite de vérifier si la suture croisée de nerfs différents était réalisable. Ces diverses recherches permirent à LANGLEY et ANDFRSON (I904) d'édicter leur règle de compatibilité qui, depuis la découverte de la médiation chimique, peut s'exprimer ainsi : il est possible de réinnerver un organe en réalisant une suture nerveuse croisée entre deux nerfs cholinergiques ou entre deux nerfs adrénergiques, mais il est impossible de remplacer un nerf cholinergique par un nerf adrénergique et réciproquement.

De nombreux travaux ont confirmé cette théorie, et en particulier la réinnervation de fibres musculaires par les fibres préganglionnaires orthosympathiques a été maintes fois réalisée (MISLAVSKY, I9O2 ; LANGLEy et ANDERSON, I904 ; HilitarP, I946). Par contre il ne semble pas qu'une étude semblable ait été effectuée pour les fibres préganglionnaires parasympathiques. Iivers auteurs ont bien provoqué la réinnervation de muscles striés par des fibres vagales, mais la suture était faite soit au cou, soit dans la région parotidienne, et le résultat obtenu pouvait être attribué à des fibres nerveuses se rendant primitivement aux muscles striés de 1'œsophage ou du larynx (Philipeaux et VUlPIAN, I863 ; REICHERT, I885 ; CalugarEanU et Henri, Igoo ; ANokhin et Iwanow, I936; Guth et Franck, I959).

Nous avons, quant à nous, réalisé une suture nerveuse vago-phrénique intrathoracique, et nous pouvons affirmer que des fibres nerveuses préganglionnaires vagales ont reconstitué au contact des fibres musculaires striées du diaphragme des jonctions neuro-musculaires fonctionnelles. Nous nous proposons d'exposer ici les arguments qui nous autorisent à émettre cette affirmation. Nous montrerons également que la suture nerveuse croisée hétérogène fournit une technique commode pour enregistrer chez l'animal éveillé l'activité unitaire des fibres efférentes vagales. Les résultats que cette technique nous a permis d'obtenir en ce qui concerne l'innervation motrice de l'estomac des Ruminants feront l'objet d'un article ultérieur.

\section{MATÉRIEL, ET MÉTHODES}

Les expériences ont été effectuées chez des moutons des deux sexes et chez des boucs castrés. Douze animaux (six moutons et six boucs) ont survécu à la série d'opérations et ont pu être utilisés pour les enregistrements.

\section{I ${ }^{\circ}$ TeChniques ChiruRgical,es}

$$
\text { A. - Anesthésie }
$$

Les animaux, à jeun depuis 36 heures, sont anesthésiés par injection intrajugulaire d'un mélange de nembutal (éthyl-méthyl-butyl barbiturate de sodium), de penthiobarbital sodique (éthyl-méthyl-butyl-thiobarbiturate de sodium) et d'atropine. Une sonde intra-trachéale à ballonnet est mise en place, et l'anesthésie est entretenue par l'inhalation d'un mélange gazeux oxygène- 
protoxyde d'azote-trichloréthylène. L'appareil d'anesthésie gazeuse, du type à pression constante et débit variable (appareil PESTY), peut être branché sur la sonde trachéale soit directement, soit par l'intermédiaire d'une pompe à respiration artificielle à expiration passive (PALMER, $500 \mathrm{~cm}^{8}$.)

\section{B. - Suture nerveuse pneumogastrique-phrénique}

Nous pénétrons dans le thorax grâce à l'incision du huitième espace intercostal. Les côtes ne sont pas réséquées, mais seulement écartées à l'aide d'un fort écarteur automatique à crémaillère. Le poumon est détaché du médiastin depuis l'arrière jusqu'au hile, et récliné vers l'avant. La section vagale porte soit sur le nerf pneumogastrique supérieur gauche un peu avant le lieu de son anastomose avec le nerf supérieur droit, soit sur la partie la plus distale du tronc vagal inférieur ( $\left.{ }^{\mathfrak{(}}\right)$. Nous sectionnons également le nerf phrénique gauche; la ligature de son extrémité centrale permet d'éviter à la fois une hémorragie gênante et la régénération intempestive du nerf. L'extrémité centrale du

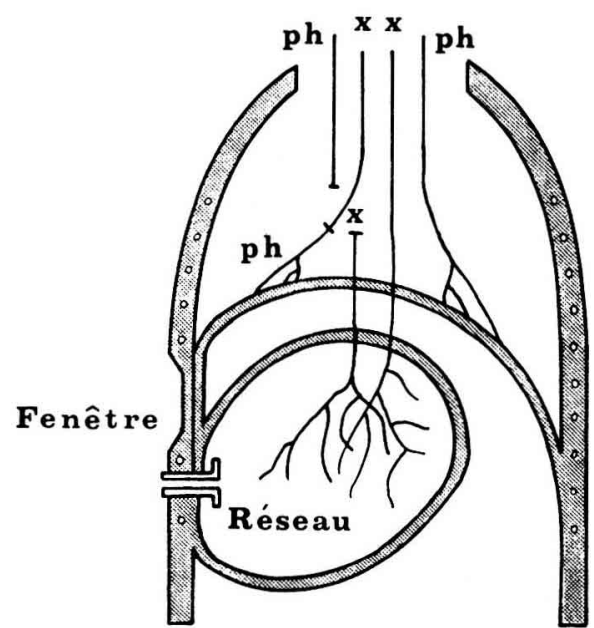

FIG. I. - Schéma d'un animal préparé pour l'enregistrement simultané des contractions du réseau et de l'électromyogramine du diaphragme réinnervé par le pneumogastrique

Noter : la suture nerveuse croisée entre le pneumogastrique (X) et le phrénique (ph), la mise du diaphragme sous la peau au niveau du sinus costo-diaphragmatique gauche (fenêtre), la fistule gastrique.

pneumogastrique et l'extrémité périphérique du phrénique sont affrontées avec un ou deux points de soie blanche très fine (aiguilles serties) qui prennent uniquement l'épinèvre (fig. I).

Il n'est pas possible de pratiquer la suture du tronc vagal supérieur (après l'union des nerfs supérieurs gauche et droit) car la section de ce tronc est très mal tolérée ; elle est le plus souvent mortelle (DUSSARDIER, I96r $b$ ).

Pour refermer le thorax, les côtes adjacentes à l'incision pariétale sont rapprochées grâce à trois fils de soie qui les enserrent. Nous suturons les muscles, le peaucier et la peau. Le vide pleural est rétabli grâce à un tube de caoutchouc branché sur une pompe à vide correctement réglée. Ce tube est laissé dans la plaie jusqu'à la fin de l'opération.

\section{C. - Mise du diaphragme "sous la peau"}

Cette opération, faite environ huit mois après la précédente, a pour but de transformer le diaphragme en muscle sous-cutané a fin de pouvoir détecter son activité électrique avec une aiguille de BRONK. Le principe général de la technique consiste à pratiquer une fenêtre dans la cage thoracique grâce à la résection de la région inférieure d'un certain nombre de côtes et des muscles intercostaux correspondants, puis à suturer le diaphragme au pourtour de la fenêtre et à la face interne

( $\left.{ }^{1}\right)$ Pour les rappels anatomiques concernant le vague intrathoracique, voir DUSSARdrer (rg62). 
de la peau (fig. 2). Nous ne pouvons pas préciser le lieu de la fenêtre, car sa position a varié chez nos divers sujets. En effet, pour que l'opération soit réalisable il faut que le sinus costo-diaphrag. matique soit pratiquement virtuel au lieu opératoire choisi, or il existe des variations individuelles d'un animal à l'autre. D'autre part pour que l'opération soit intéressante il faut que la région du diaphragme mise sous la peau soit réinnervée, et là encore existent đes différences individuelles. En pratique, nous commençons par inciser la peau le long de la neuvième côte, nous effectuons la résection sous-périostée de cette côte et nous pénétrons dans le thorax en incisant le lit périosté ; c'est alors que nous choisissons l'emplacement de la fenêtre. A la fin de l'opération, il faut rétablir le vide pleural, sinon l'air pénètre entre le diaphragme et la peau et gêne la cicatrisation. Ce vide est rétabli au moyen d'une sonde de caoutchouc introduite dans le thorax par une ponction spéciale d'un espace intercostal.

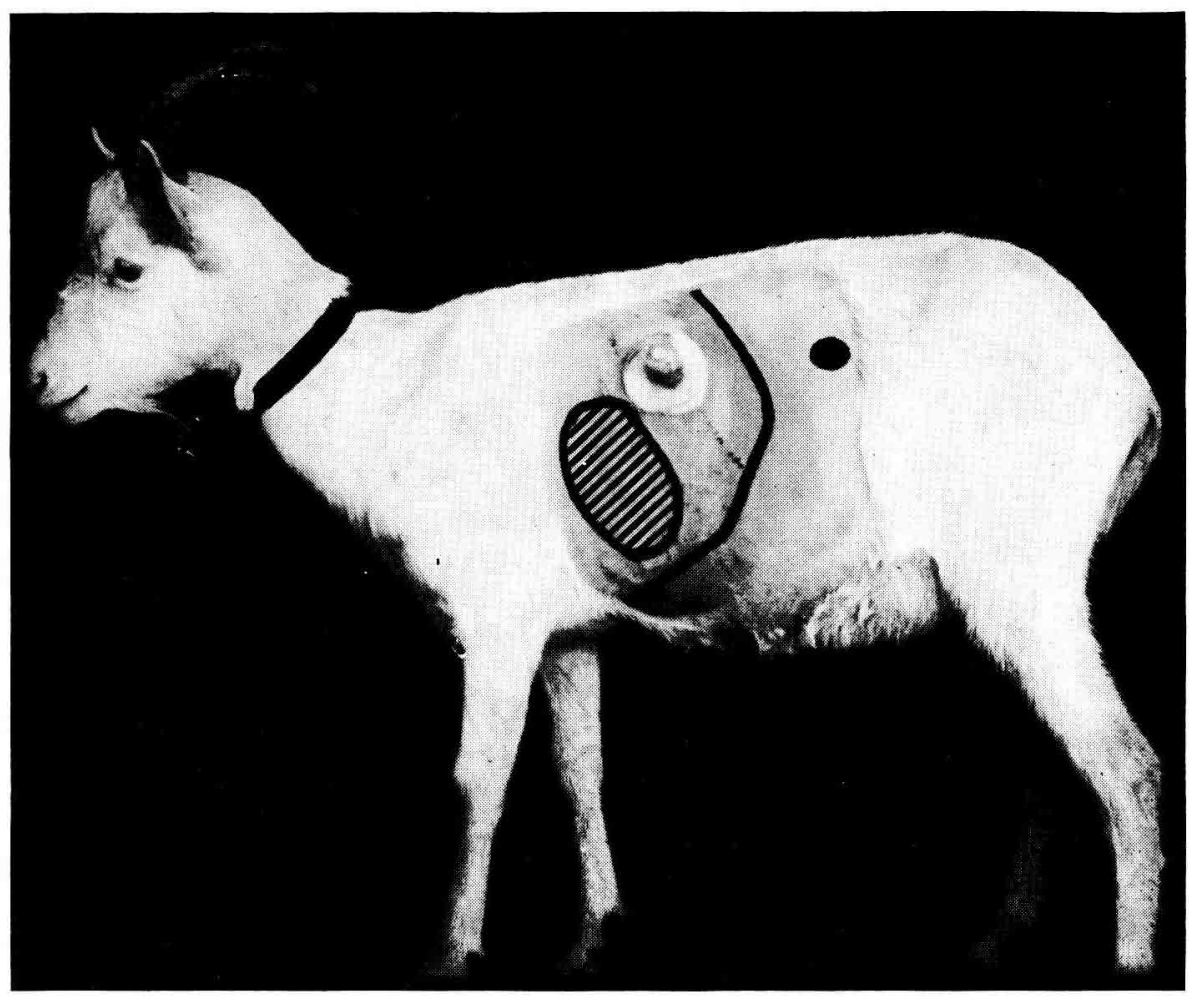

FIG. 2. - Bouc muni d'une fistule de la région antérieure du rumen

Remarquer la canule en plexiglass. La ligne noire représente le cercle de l'hypochondre. Le point noir indique l'endroit où se fait normalement la fistule du rumen. Les hachures délimitent la zone du thorax où le diaphragme peut être mis sous la peau.

\section{D. - Fistule de la région antérieure du rumen}

Afin de permettre l'introduction dans le réseau (deuxième poche gastrique) d'un ballon destiné à en enregistrer les contractions, les animaux ont été munis d'une fistule de la région antérieure du rumen (fig. 2). Pour atteindre cet organe, situé en région sous-costale, nous avons utilisé une voie d'accès transthoracique. Le détail de la technique a déjà été publié (DussardiER, ig6i a). Cette opération était effectuée un mois environ après la mise du diaphragme " sous la peau ». 


\section{0) TEChNiques D'ENREgistrement}

Les animaux ont été examinés une ou deux fois par semaine. Ils n'étaient pas anesthésiés, mais simplement attachés à l'aide d'un collier et d'une chaîne dans une stalle ouverte du côté de l'expérimentateur.

\section{A. - Réception, amplification et enregistrement des potentiels électromyographiques}

L'électromyogramme du diaphragme a été capté à l'aide d'aiguilles coaxiales dites aiguilles de Bronk (ADRIAN et BRONK, I928), ayant un seul fil central ; le corps de l'aiguille était verni. Pour l'amplification des potentiels recueillis nous avons utilisé des amplificateurs à lampes à couplage par capacité - résistance de type courant en électrophysiologie. Dans certains cas les amplificateurs étaient précédés d'un étage "changeur d'impédance ". L'enregistrement des potentiels était réalisé par la photographie de l'écran d'un oscillographe cathodique à deux spots (Cossor IO49 mk III).

\section{B. - Enregistrement des pressions intragastriques}

Un ballon enregistreur introduit dans le réseau à travers la fistule gastrique transmettait les variations de pression à un capteur électronique à capacité variable (appareil RACIA), qui les transformait en différences de potentiel. Celles-ci pouvaient alors être enregistrées en même temps que l'électromyogramme, grâce à l'oscillographe cathodique.

\section{$3^{\circ}$ ) TeChNiques histologiques}

Les animaux ayant servi aux enregistrements ont été ensuite sacrifiés et diverses pièces anatomiques ont été prélevées aux fins de contrôles histologiques.

$$
\text { A. - Techniques de coloration }
$$

Nous n'avons utilisé que des techniques histologiques courantes :

Pour les nerfs : coloration de la myéline par immersion pendant huit jours dans une solution d'acide osmique à I p. Ioo.

Pour la paroi gastrique : trichrome de Masson, imprégnation argentique en bloc de CAJALd'ANCONA.

Pour le diaphragme : trichrome de Masson, imprégnation argentique en bloc de CAJAL-d'ANCONA, hématoxyline phosphomolybdique au dioxane de Trovas (I943) modifiée par DUMONT et PARIS (1959).

\section{B. - Dépouillement des résultats}

Le dépouillement quantitatif de nos pièces histologiques a été réalisé en grande partie grâce à des méthodes de projection.

Pour la mesure du diamètre des fibres musculaires du diaphragme, les coupes ont été projetées sur un papier calque et dessinées. Etant donné la grande variation de forme de la section des fibres musculaires, il nous a paru difficile de faire directement une mesure de diamètre satisfaisante. Nous avons donc découpé le contour de chacune des fibres, estimé la surface par pesée, et calculé le diamètre d'un cercle de même surface.

La mesure du diamètre des fibres nerveuses ne présente pas les mêmes difficultés, car la forme s'éloigne peu du cercle. Nous avons projeté la coupe sur un papier calque et dessiné le contour des fibres. Puis, pour avoir plus de précision, les dessins ont été projetés à leur tour à l'aide d'un agrandisseur photographique; nous avons alors évalué avec un gabarit comportant des cercles de diverses dimensions, le diamètre des différentes fibres.

Le système de projection utilisé pour les fibres nerveuses (fort grossissement) a été mis au point par M. VERGÉ (ingénieur à l'I. N. R. A.) ; celui utilisé pour la projection des fibres musculaires (grossissement moyen) a été conçu et réalisé par M. Rougeot (maître de recherches à l'I. N. R. A.). 


\section{RÉSULTATS}

\section{Io) ÉTUDES ÉLECTROPHYSIOLOGIQUES}

\section{A. - Envegistrement del'activité électromyographique spontanée du diaphragme réinnervé}

Si nous recueillons l'électromyogramme d'un diaphragme mis sous la peau après qu'il ait été séparé du phrénique et réinnervé par les fibres vagales, nous constatons que son activité est généralement rythmée soit sur les contractions œsophagiennes (déglutition) soit sur les contractions gastriques; nous ne trouvons pas d'activité de type respiratoire, contrairement à ce qui se passe pour un diaphragme doté de son innervation normale. Les activités " de déglutition " ne nous intéressent pas ici (elles feront l'objet d'une autre publication) car elles traduisent manifestement la réinnervation du diaphragme par des fibres vagales qui se rendaient primitivement à l'œsophage, dont la musculeuse est constituée par des fibres striées. Par contre les activités rythmées sur les contractions gastriques ont directement trait au sujet.

La figure 3 montre un enregistrement obtenu chez un bouc I2 mois après l'exécution de la suture vago-phrénique. La première ligne des figures $\mathrm{A}, \mathrm{B}$ et $\mathrm{C}$ et la figure D représentent l'électromyogramme d'une même unité motrice (enregistrée à des vitesses de déroulement croissantes). La deuxième ligne des figures $\mathrm{A}, \mathrm{B}$ et $\mathrm{C}$ correspond à l'enregistrement des pressions à l'intérieur du réseau. Le ballon enregistre évidemment des variations de pression de faible amplitude dues à la respiration, mais il enregistre également des variations de plus grande amplitude qui traduisent les contractions biphasiques du réseau provoquées par le vague droit resté en place. Nous voyons que 1'unité considérée, qui est inactive pendant la période de repos de l'estomac, pulse pendant la première phase de la contraction du réseau.

Des salves de potentiels liées chronologiquement à la motricité gastrique sont recueillies assez facilement dans un diaphragme réinnervé par le vague. Le rapport temporel existant entre la contraction et l'électromyogramme est stable pour une unité donnée, mais varie d'une unité à l'autre. Ceci n'est pas surprenant car nous pouvons recueillir, outre l'activité des fibres motrices se rendant primitivement au réseau, celle des fibres se rendant à différentes régions de l'appareil gastrique qui entrent en activité après le réseau. Ce problème sera plus spécialement étudié dans un article ultérieur.

Retenons simplement l'existence d'un rapport chronologique entre l'activité électromyographique du diaphragme et la motricité gastrique.

\section{B. - Morphologie des potentiels électromyographiques spontanés}

Lorsque l'électromyogramme du diaphragme est enregistré à faible vitesse les potentiels apparaissent comme des spikes brèves. En réalité, sur les enregistrements à grande vitesse, on s'aperçoit qu'ils ont une morphologie variable et complexe : ils sont souvent polyphasiques (fig. 4) et peuvent durer 30 millisecondes. 
Il s'agit bien à chaque fois de l'activité d'une seule unité car la forme peut rester stable au cours des pulsations successives.

En principe la forme du potentiel donné par une unité motrice doit être constante dans le temps. En se basant sur ce critère, on peut savoir si l'on enregistre une seule unité motrice ou plusieurs, et dans ce dernier cas, on peut les distinguer entre elles et préciser leurs paramètres respectifs. Dans la pratique le dépouillement des films s'est révélé laborieux. Nous avons dî arriver à la conclusion que les potentiels
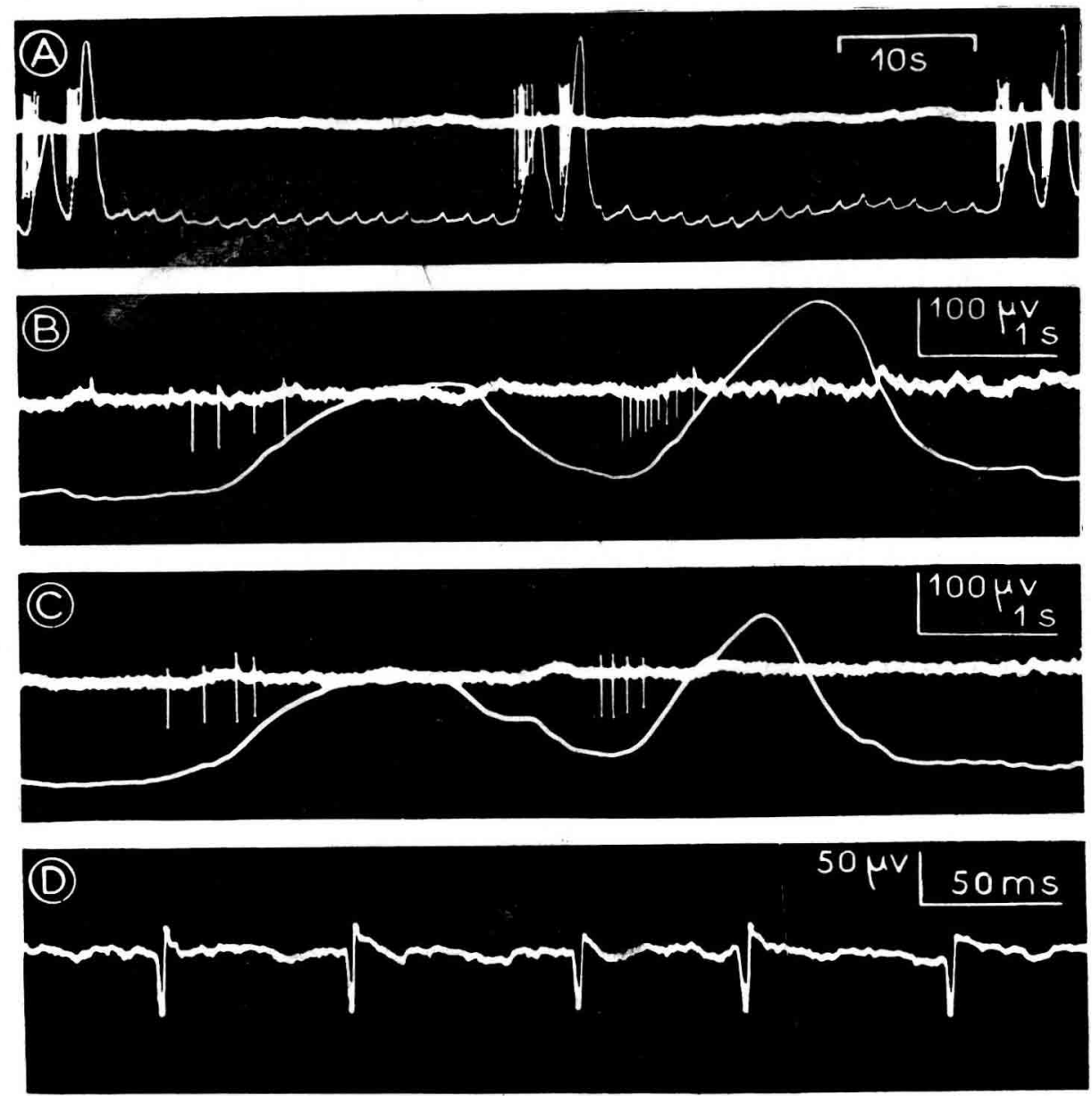

FIG. 3. - Électromyogramme d'un diaphragme de bouc réinnervé par le vague et mécanogramme du réseau.

La première ligne des figures $\mathrm{A}, \mathrm{B}$ et $\mathrm{C}$, et la figure $\mathrm{D}$ représentent l'EMG d'une même, unité motrice enregistré à des vitesses de déroulement croissantes $(t=10 \mathrm{~s}, t=\mathrm{I} \mathrm{s}, t=50 \mathrm{~ms})$. Etalonnage d'amplitude pour l'EMG : roo et $50 \mu \mathrm{V}$. La deuxième ligne des figures $\mathrm{A}, \mathrm{B}$ et $\mathrm{C}$ correspond à l'enregistrement de la contraction du réseau. Remarquer :

- sur la figure A que l'unité motrice pulse seulement avant chacune des deux contractions du réseau.

Elle ne présente ni activité respiratoire, ni activité tonique.

- sur les figures B et C que l'activité électromyographique précède la contraction;

- sur la figure D que le potentiel d'action unitaire est sensiblement biphasique.

Annales de Biologie animale. - 1962. 
successifs donnés par une même unité ne sont pas toujours identiques (fig. 4). Il peut arriver que l'amplitude subisse des fluctuations importantes, ou que certains éléments du potentiel polyphasique n'apparaissent que par intermittence, ou encore que toutes les pointes s'extériorisent mais que leur amplitude respective varie. Nous avons essayé de comprendre l'origine de ces fluctuations.

La variation de la forme du potentiel peut traduire simplement les déplacements de l'aiguille de réception sous l'influence de la contraction musculaire. Le diagnostic
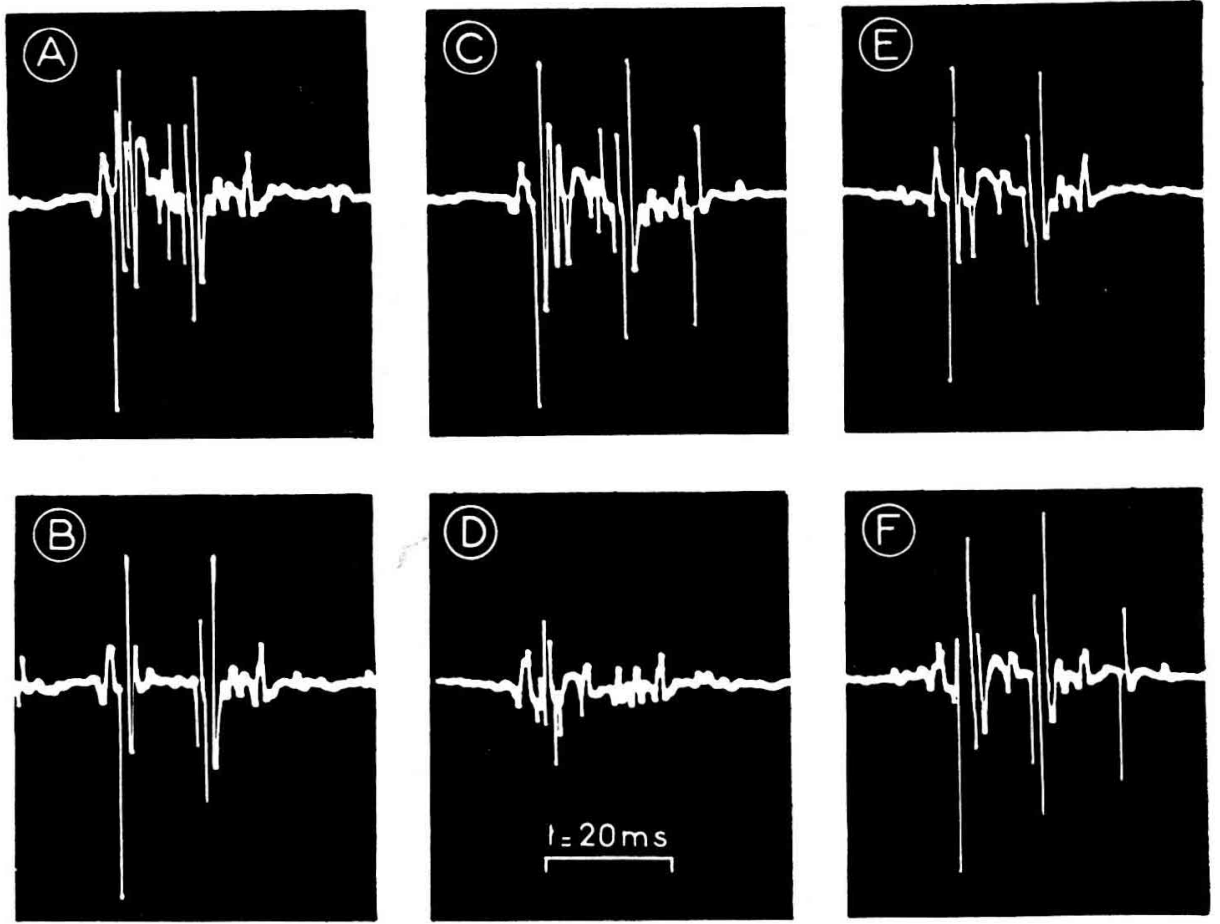

Fic. 4. - Iiluctuation de la forme des potentiels de rétnnervation

Diaphragme de mouton réinnervé par le pneumogastrique. La figure représente six potentiels d'action successifs d'une même unité motrice pulsant spontanément.

est évident lorsque la déformation est définitive ; il est plus difficile lorsque l'aiguille, après avoir été déplacée, revient ensuite à sa place primitive.

A côté de cette cause de variation banale il en est une autre plus intéressante : dans certains cas divers éléments de la réponse polyphasique disparaissent par moments, sans que le reste de la réponse soit altéré. Le phénomène est très net lorsqu'il intéresse les pointes les plus amples. Il est arrivé que les premiers potentiels d'une salve aient une certaine morphologie stable, et que, à la fin de la salve, certaines pointes n'apparaissent qu'une fois sur deux. Le phénomène se reproduisait à chaque salve. Il ne semble pas que ce puisse être un artéfact de réception : il y avait manifestement une absence d'activité de certaines des sources. 


\section{C. - Contraction diaphragmatique produite par la stimulation électrique du pneumogastrique}

Chez l'animal anesthésié, nous avons placé deux électrodes de stimulation sur le nerf pneumogastrique, l'une au niveau cervical, l'autre dans le thorax, en amont du lieu de la suture avec le phrénique. L'électromyogramme du diaphragme mis à nu a été recueilli soit en dérivation monopolaire, soit en dérivation bipolaire, avec une aiguille de BRONK. La stimulation du vague produit une contraction du diaphragme, visible à l'œil nu, et décelable par la technique électromyographique.
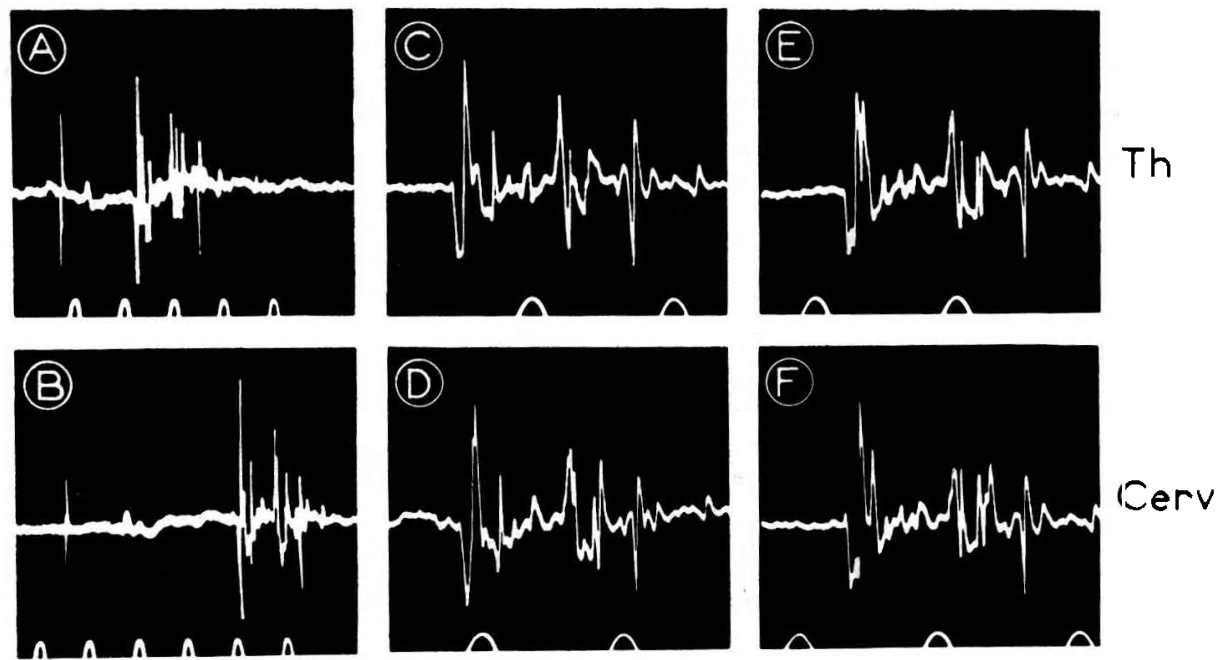

FIG. 5. - Électromyogramme d'un diaphragme de bouc réinneréé par le pneumogastrique

Les contractions diaphragmatiques sont provoquées par la stimulation électrique du nerf dans le thorax pour les figures $\mathrm{A}, \mathrm{C}$ et $\mathrm{E}$; au niveau cervical pour les figures $\mathrm{B}, \mathrm{D}$ et $\mathbf{F}$.

En A et $B$ on a l'ensemble de la réponse. L'artéfact de stimulation est visible sur la gauche des figures. Noter l'existence d'un petit potentiel biphasique précoce (première unité) et d'un potentiel polyphasique plus tardif (deuxième unité). La latence et l'écart de ces deux potentiels sont plus grands avec une stimulation cervicale quavec une stimulation thoracique (longueur de conduction respective 16 et 50 centimètres).

En C, D, E, F, on voit seulement le potentiel polyphasique. Noter que sa forme ne dépend pas de la longueur de conduction.

Temps : 50 cycles par seconde.

L'électromyogramme ainsi recueilli est apparu le plus souvent polyphasique. Dans ce cas évidemment, l'interprétation est moins directe que pour les potentiels spontanés, car on peut penser que l'on enregistre l'activité de plusieurs unités motrices dont les fibres nerveuses ont des vitesses de conduction différentes. Mais nous avons vérifié, en nous plaçant au seuil de stimulation, que la réponse globale répondait à la loi du tout ou rien. Bien mieux, nous avons pu montrer que la forme du potentiel ne se modifiait pas lorsque le nerf était stimulé à deux endroits éloignés l'un de l'autre (dans le cas de la figure 5 les longueurs de conduction nerveuse étaient respecti- 
vement de 16 et $50 \mathrm{~cm}$ environ). Enfin dans certains cas nous avons pu vérifier en stimulant le nerf non sectionné, que les potentiels spontanés et ceux produits par la stimulation électrique du nerf avaient la même forme.

Dans des conditions où nous étions certain, d'après les critères précédents, de recueillir l'activité d'une seule unité motrice, nous avons retrouvé les fluctuations de la forme des potentiels d'action polyphasiques déjà observées avec les potentiels spontanés (fig. 6).
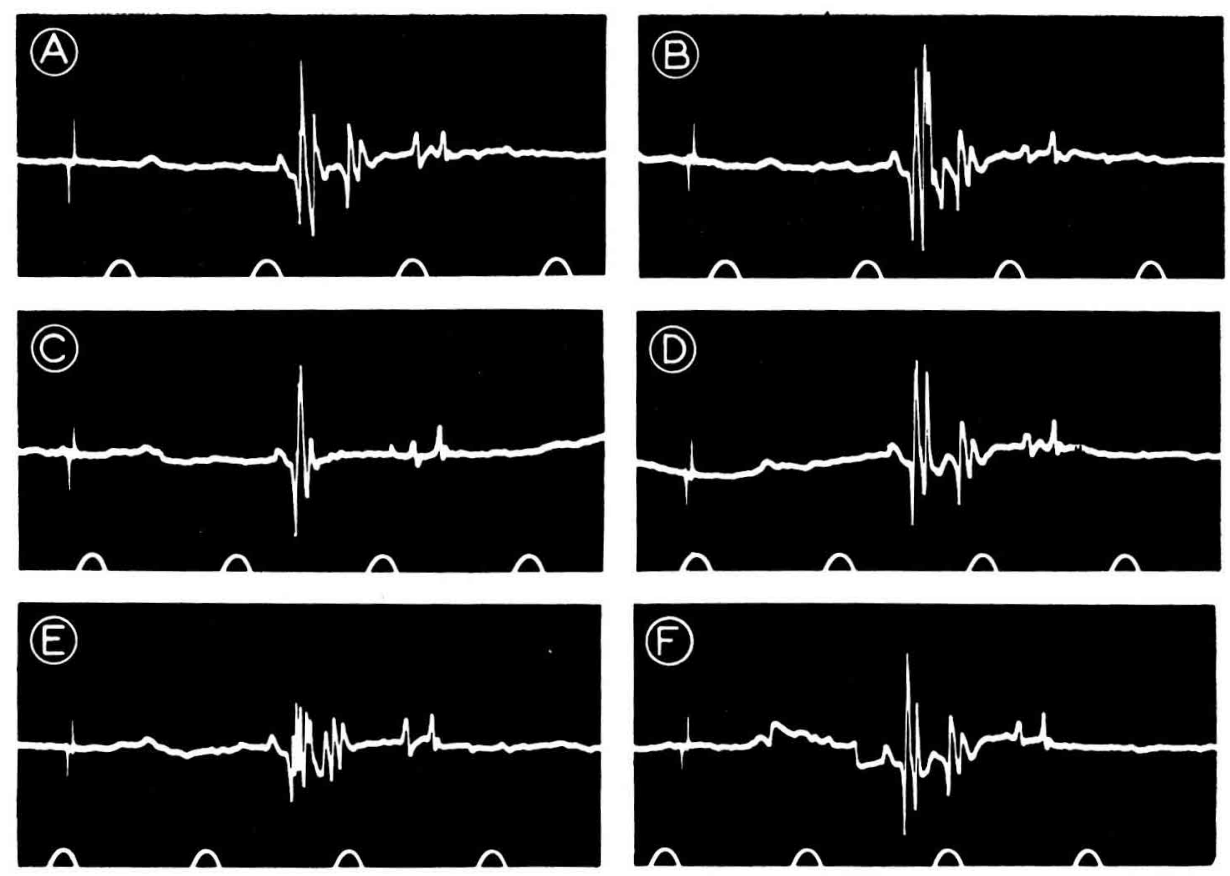

FIG. 6. - Électromyogramme d'un diaphragme de bouc réinnevé par le pneumogastrique

Les contractions sont provoquées par la stimulation électrique du vague à la fréquence de io chocs par seconde. La figure représente six réponses successives de la même unité motrice. Noter les fluctuations de la forme du potentiel polyphasique.

Temps : 50 cycles par seconde.

La stimulation électrique nous a permis de calculer la vitesse de conduction des fibres nerveuses. En nous basant sur les différences des latences des réponses obtenues avec les deux stimulations électriques : la cervicale et la thoracique, nous avons trouvé pour différentes unités motrices des vitesses de $2-2,6-3, \mathrm{I}-4,6-6,7-9,8$ I6,5 - I8,8 - 24,6 et 29,5 mètres par seconde. Lorque nous avons essayé de calculer la vitesse de conduction en nous basant simplement sur la latence d'une réponse, nous avons trouvé des valeurs plus faibles et qui étaient plus petites avec une stimulation thoracique qu'avec une stimulation cervicale. Ceci s'explique par un ralentissement de la conduction dans la partie terminale des fibres. L'erreur faite en ne tenant compte que d'une latence est manifeste d'après les exemples du 
tableau I. Elle est particulièrement grande dans le cas de 1'unité $\mathrm{n}^{\circ} 4$; pourtant le calcul montre qu'il suffit d'admettre l'existence à l'extrémité de la fibre nerveuse

TABLEAU I

Vitesse de conduction de cinq fibres vagales efférentes qui ont ríinnervé le diaphragme
à la suite d'une suture nerieuse croisée v'ago-phrénique

Nous avons stimulé le vague soit an niveau cervical, soit dans le thorax et mesuré le temps de latence de la contraction diaphragmatique. Nous pouvons calculer la vitesse de conduction des fibres de trois manières différentes. I.es valeurs domnées par les trois méthodes ne concordent pas, ce qui prouve l'existence d'um ralent issement important de la vitesse de conduction dans les portions terminales des fibres.

\begin{tabular}{|c|c|c|c|}
\hline \multirow{2}{*}{$\begin{array}{l}\text { Unité motrice } \\
\qquad N^{\top}\end{array}$} & \multicolumn{2}{|c|}{ Vitesse calculée d'après la latence (en mètres par seconde) } & \multirow{2}{*}{$\begin{array}{c}\text { Vitesse calculée d'après la } \\
\text { différence des latences (en } \\
\text { mètres par seconcle) }\end{array}$} \\
\hline & Stimulation thoracique & Stimulation cervicale & \\
\hline 1 & j & 6 & 6,7 \\
\hline 2 & 7 & 8,7 & 9,8 \\
\hline 3 & 13 & $1: t, 9$ & 16,5 \\
\hline 4 & 6,2 & 10,8 & 18,8 \\
\hline 5 & 17,5 & 21,7 & 24,6 \\
\hline
\end{tabular}

d'une portion amyélinique de $8,5 \mathrm{~mm}$ de long, ayant une vitesse de conduction de 0,6 mètre par seconde, pour expliquer les vitesses obtenues.

\section{2. 一 CONTRÔLES HISTOLOGIQUES}

Nous avons étudié le tronc nerveux, le muscle et les fibres nerveuses dans leur trajet intra-musculaire. L'ancienneté de la suture variait selon les pièces de 8 mois à 3 ans.

\section{A. - Étude du phrénique réinnervé}

Le spectre de diamètre des fibres myélinisées d'un nerf phrénique normal a une allure grossièrement bimodale. Chez un bouc (fig. 7) un nerf gauche comprenait environ I 800 fibres, dont 430 de I à $8 \mu$, et I 370 de 9 à $I_{7} \mu$. Chez un mouton nous avons trouvé 2340 fibres, dont 800 de I à $8 \mu$, et I 540 de 9 à $20 \mu$.

Dans un nerf phrénique réinnervé par des fibres du pneumogastrique, toutes les fibres ont un diamètre compris entre I et $7,5 \mu$, avec un maximum pour $3 \mu$ (fig. 7 ). Chez deux moutons, nous avons trouvé respectivement I 320 et 3 Ioo fibres ; chez un bouc nous en avons trouvé I 2 I5. Dans l'ensemble, les fibres que l'on retrouve dans un phrénique réinnervé ressemblent beaucoup plus à celles du vague qu'à celles d'un phrénique normal (fig. 7). Cependant, leur spectre de diamètre est un peu différent de celui des fibres vagales normales. Le mode est un peu plus élevé (voir tableau 2). 


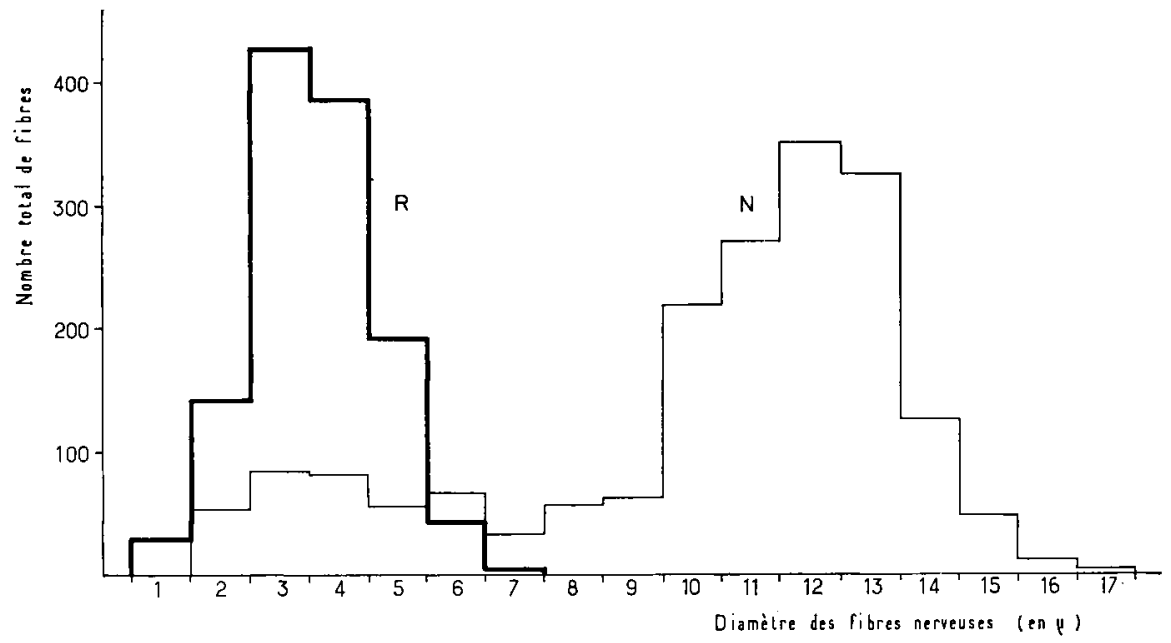

FIG. 7. - Spectre de dianètre des fibres nerveuses d'un phrénique gauche suturé avec le vague (R) et du phrénique droit normal (N) du même animal (bouc)

Le nombre de libres (orclonnée) est porté en valeur absolue. Les deux spectres sont nettement différents.

TABIEAU 2

Nombre de fibres myélinisées de différents diamètres trouvées chez des moutons dans un pneumogastrique normal (nerf vague thoracique supérieur gauche), dans un phrénique normal et dans deux phréniques réinnervés par des fibres vagales (suture croisce entre le nerf vague thoracique supérieur gauche et le phrénique homo-latéral)

\begin{tabular}{|c|c|c|c|c|c|c|c|c|}
\hline \multirow{3}{*}{$\begin{array}{c}\text { Diametre } \\
\text { des } \\
\text { fibres } \\
(\mu)\end{array}$} & \multirow{2}{*}{\multicolumn{2}{|c|}{$\begin{array}{c}\text { Pneunogastrique } \\
\text { normal }\end{array}$}} & \multicolumn{4}{|c|}{ Phrénique réinnervé } & \multirow{2}{*}{\multicolumn{2}{|c|}{ Phrénique normal }} \\
\hline & & & Mouton & 6020 & Mouton & 6002 & & \\
\hline & $\begin{array}{l}\text { Nombre de } \\
\text { fibres }\end{array}$ & $\begin{array}{l}\text { Pourcen- } \\
\text { tage }\end{array}$ & $\begin{array}{c}\text { Nombre de } \\
\text { fibres }\end{array}$ & $\begin{array}{l}\text { Pourcen- } \\
\text { tage }\end{array}$ & $\begin{array}{l}\text { Nombre de } \\
\text { fibres }\end{array}$ & $\begin{array}{l}\text { Pourcen- } \\
\text { tage }\end{array}$ & $\begin{array}{c}\text { Nombre de } \\
\text { fibres }\end{array}$ & $\begin{array}{c}\text { Pourcen- } \\
\text { tage }\end{array}$ \\
\hline 1 & 330 & 2,7 & 123 & 4,0 & 74 & 5,6 & 9 & 0,4 \\
\hline 2 & 1304 & 35,0 & 620 & 20,0 & 320 & 24,3 & 113 & 4,8 \\
\hline 3 & 5608 & 45,1 & 1237 & 40,0 & 389 & 29,5 & $23^{\prime} t$ & 10,0 \\
\hline f & 1561 & 12,7 & 786 & $25,4^{\prime}$ & $33^{\prime} 7$ & $26, t^{\prime}$ & 151 & 6,4 \\
\hline 5 & 317 & 2,6 & 235 & 7,6 & 1168 & 11,3 & 108 & 4,6 \\
\hline 6 & 123 & 1,0 & 80 & 2,6 & 39 & 2,9 & 102 & 4,5 \\
\hline 7 & 50 & 0,4 & 11 & 0,4 & 0 & 0 & 50 & 2,1 \\
\hline 8 & 16 & 0,13 & 0 & 0 & 0 & 0 & 38 & 1,6 \\
\hline 9 & 6 & 0,05 & 0 & 0 & 0 & 0 & 38 & 1,6 \\
\hline 10 & 3 & 0,02 & 0 & 0 & 0 & 0 & 93 & 4,0 \\
\hline 11 & 0 & 0 & 0 & 0 & 0 & 0 & 120 & 5,1 \\
\hline 12 & 0 & 0 & 0 & 0 & 0 & 0 & 129 & 5,5 \\
\hline 13 & 0 & 0 & 0 & 0 & 0 & 0 & $28:$ & 12,0 \\
\hline $11 \mathrm{t}$ & 0 & 0 & 0 & 0 & 0 & 0 & 372 & 15,9 \\
\hline 15 & 0 & 0 & 0 & 0 & 0 & 0 & 306 & 13,1 \\
\hline 16 & 0 & 0 & 0 & 0 & 0 & 0 & 127 & 5,4 \\
\hline 17 & 0 & 0 & 0 & 0 & 0 & 0 & 50 & 2,1 \\
\hline 18 & 0 & 0 & 0 & 0 & 0 & 0 & 14 & 0,6 \\
\hline 19 & 0 & 0 & 0 & 0 & 0 & 0 & 5 & 0,2 \\
\hline 20 & 0 & 0 & 0 & 0 & 0 & 0 & 2 & 0,1 \\
\hline Total & 12318 & 100 & 3092 & 100 & 1317 & 100 & 2343 & 100 \\
\hline
\end{tabular}




\section{B. - Etude du muscle réinnervé}

Au simple examen visuel, les hémi-diaphragmes réinnervés par les fibres vagales paraissent nettement atrophiés par rapport aux hémi-diaphragmes normaux correspondants. Ils sont moins épais, plus pâles, et contiennent souvent des traînées blanches, parallèles aux fibres musculaires; ce sont manifestement des zones dans lesquelles le tissu musculaire a été remplacé par du tissu conjonctif. L'atrophie est beaucoup plus marquée chez les boucs que chez les moutons. Sur des coupes histologiques perpendiculaires à la direction des fibres, on est frappé tout d'abord par la quantité considérable de tissu conjonctif qui sépare les faisceaux de fibres et même les fibres d'un même faisceau ; il existe également de nombreuses zones claires correspondant à des traînées de tissu adipeux enlevé par les solvants organiques utilisés pour l'inclusion à la paraffine; on est donc en présence d'une surcharge fibro-adipeuse. Le deuxième point remarquable à l'examen microscopique est le calibre des fibres musculaires. La figure 8 (A et $B$ ) montre que le diamètre moyen et le diamètre maximum des fibres sont beaucoup plus élevés dans l'hémi-diaphragme réinnervé par le pneumogastrique que dans l'hémi-diaphragme opposé. On trouve des fibres géantes ayant un diamètre de roo $\mu$.

Lorsqu'un diaphragme a été réinnervé par son propre nerf, celui-ci ayant été sectionné et resuturé immédiatement, on n'observe pas les mêmes 'phénomènes, bien que la suture soit faite à la même distance du muscle. A l'examen macroscopique, le côté réinnervé paraît semblable au côté normal ; on constate également peu d'altérations histologiques. On note simplement un léger épaississement des gaines conjonctives. Le spectre de diamètre des fibres paraît modifié ; il est peutêtre légèrement décalé vers la gauche (fig. $8 \mathrm{C}$ et $\mathrm{D}$ ), ce qui indiquerait une légère atrophie des fibres musculaires.

A la suite de la section du phrénique, le diaphragme s'atrophie. Chez un mouton, après deux mois de dégénérescence, nous avons calculé que les fibres avaient un diamètre moyen de $\mathrm{rx} \mu$; les dimensions extrêmes étaient de 8 et $26 \mu$. Pour 3 diaphragmes normaux nous avons trouvé des diamètres moyens de 24,26 et $4 \mathrm{I} \mu$, les diamètres minima étant respectivement de Io, I2 et $20 \mu$ et les diamètres maxima de $40,4^{\circ}$ et $60 \mu$.

\section{C. - Etude des fibres nerveuses dans leur trajet intramusculaire}

Cette étude, qui n'a encore qu'un caractère préliminaire, a été effectuée par $R$. Couteaux et J. Taxi. Nous les remercions vivement d'avoir bien voulu nous autoriser à en publier les résultats.

Les fibres nerveuses réinnervant une moitié du diaphragme après suture vagophrénique ont été mises en évidence à l'aide de la technique d'imprégnation argentique de BIELschowsky-Gros. Cette technique neurofibrillaire a montré que dans la réinnervation du diaphragme obtenue dans ces conditions, il n'y a pas seulement pénétration à l'intérieur du muscle d'un contingent important de fibres nerveuses, mais que se constituent également de véritables jonctions neuro-musculaires méritant le nom de plaques motrices.

Dans les diaphragmes de boucs ou de moutons étudiés, deux types d'innerva- 

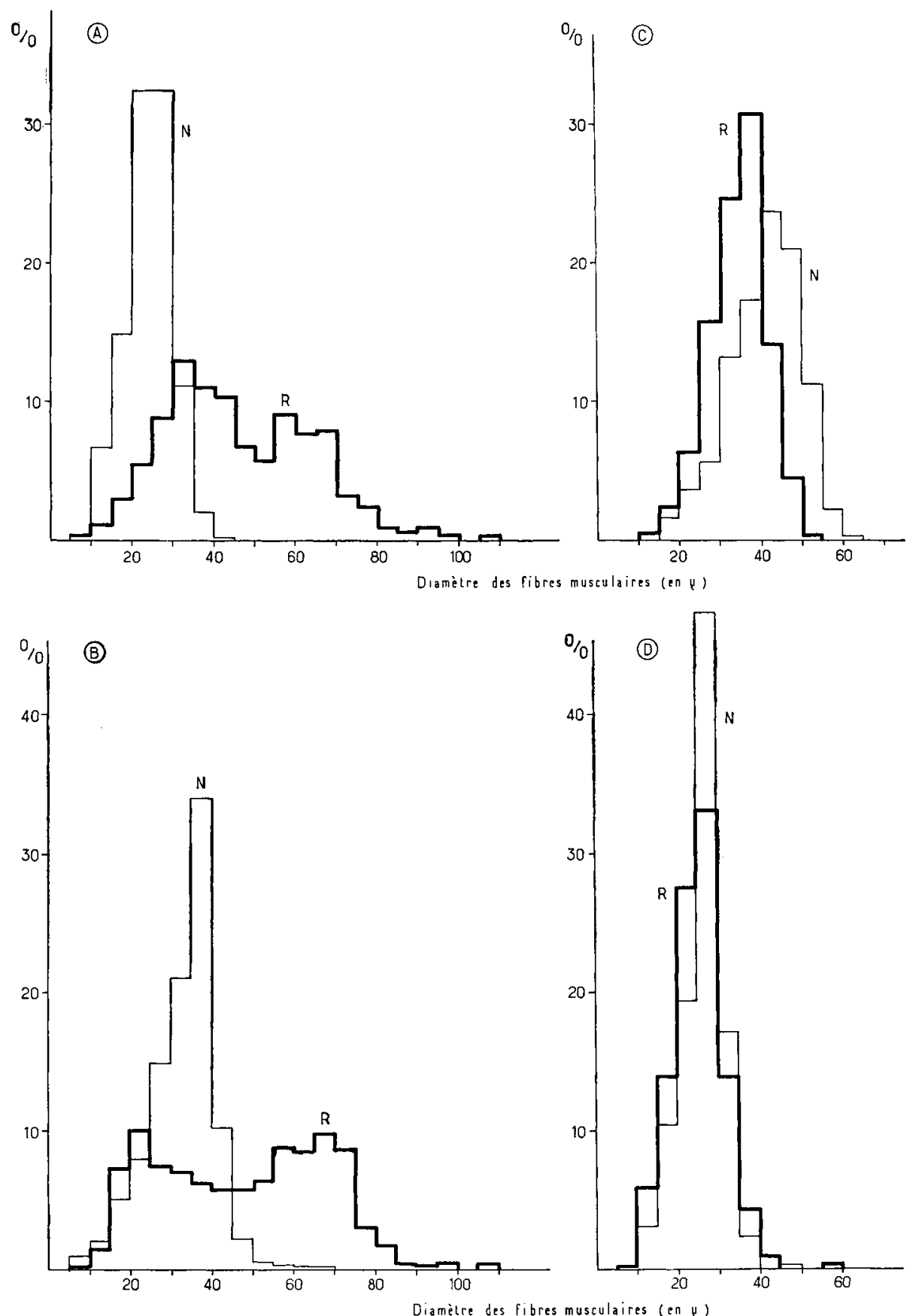

FIG. 8. - Comparaison du spectre de diamètre des fibres musculaires d'un diaphragme normal et d'un diaphragme réinnervé

Pour chacune des quatre figures les numérations ont été faites sur les deux hémi-diaphragmes d'un même animal : le gauche était réinnervé $(\mathrm{R})$; le droit était normal $(\mathrm{N})$. Le diaphragme avait été réinnervé par le vague dans le cas des figures $A$ et $B$, par son propre nerf pour les figures $C$ et $D$. L'animal d'expérience était le mouton en $A, C$ et $D$, le bouc en $B$. 
tion motrice ont été observés, dont l'importance respective et la distribution variaient considérablement d'un diaphragme à l'autre. De ces deux types d'innervation, l'un paraît en tous points conforme à l'innervation normale du diaphragme ; l'autre en diffère au contraire par un certain nombre de caractères dont nous indiquerons ci-dessous les plus notables.

Contrairement à ce qu'on observe dans un diaphragme normal, où la majeure partie des fibres nerveuses motrices ne perdent leur gaine de myéline qu'au voisinage de leur terminaison, les fibres de ce second type d'innervation se montrent, dans leur très grande majorité, dépourvues de myéline sur toute l'étendue de leur trajet intramusculaire. Ce trajet lui-même apparaît inhabituel, les faisceaux de fibres nerveuses courant souvent sur une grande longueur à la surface de fibres musculaires et parallèlement à leur axe, avant de s'articuler avec elles au niveau des plaques motrices. Enfin, les plaques motrices, elles aussi, diffèrent sensiblement des plaques motrices normales par l'extrême finesse que présentent généralement les ramifications terminales des fibres nerveuses. La détection histochimique des activités cholinestérasiques à l'aide de la méthode de KOELL niveau de ces plaques motrices, pourvues de ramures nerveuses grêles, que des activités beaucoup plus faibles qu'à l'ordinaire.

\section{DISCUSSION}

\section{I. - DONNÉES HISTOLOGIQUES}

\section{A. - Étude du phrénique réinnervé}

Selon divers auteurs (HAMmond et HiNSEY, I945; BERRY et HINSEY, I946) les fibres nerveuses de gros diamètre qui régénèrent dans un nerf habité auparavant par des fibres de petit diamètre repoussent fines mais l'inverse n'est pas vrai. Il est donc normal que les fibres trouvées dans un phrénique réinnervé par des fibres vagales ressemblent à celles du vague. On peut être surpris par contre, de voir que le spectre de diamètre est un peu décalé vers la droite. Contrairement aux conclusions des auteurs cités ci-dessus, les fibres fines auraient-elles tendance à s'élargir en régénérant dans un nerf précédemment occupé par les fibres de gros diamètre? Une sélection s'est-elle produite, les fibres de plus gros diamètre ayant régénéré mieux que les autres? Les fibres les plus fines ont-elles perdu leur myéline? Il nous paraît difficile de choisir entre ces différentes hypothèses. D'ailleurs la différence est faible et seule une étude statistique approfondie pourrait nous dire si elle est statistiquement significative.

Comme le montre le tableau 2, le nombre de fibres de chaque diamètre trouvé dans un phrénique réinnervé n'est pas supérieur à celui qui existe dans un pneumogastrique normal, mais est souvent supérieur à celui qui existe dans le phrénique contralatéral. Par conséquent, les fibres trouvées dans le phrénique réinnervé ne peuvent pas être les fibres les plus fines du phrénique : l'histologie confirme la physiologie : il y a bien eu envahissement du phrénique par les fibres vagales. 


\section{B. - Étude du muscle réinnervé}

A. - La suture vago-phrénique permet incontestablement une réinnervation des fibres musculaires du diaphragme. Cependant, bien que le nombre de fibres nerveuses qui régénèrent dans le phrénique soit égal ou supérieur au nombre de fibres qui existent normalement dans un phrénique, la récupération du muscle est loin d'être totale, contrairement à ce qui se passe lorsqu'on réalise une suture phréno-phrénique. Malgré son insuffisance, la réinnervation n'est pas moins remarquable si l'on songe que les fibres vagales, à l'exception de celles qui innervaient l'œsophage, sont des fibres préganglionnaires qui s'articulaient primitivement avec des cellules nerveuses.

B. - Il est curieux que les fibres musculaires réinnervées par le vagute subissent une hypertrophie. Ce n'est pas un artéfact, il ne s'agit pas de fibres en cours de dégénérescence dont les myofibrilles seraient fragmentées et réunies en amas, car les coupes longitudinales montrent que les fibres ont un diamètre régulier, et que l'écartement des striations est normal. La courbe de fréquence des diamètres ayant un aspect bimodal (fig. 8), nous nous étions demandé s'il n'y avait pas deux populations de fibres musculaires réinnervées par exemple, les unes par les fibres nerveuses allant primitivement au muscle strié œsophagien, les autres par les fibres préganglionnaires. On aurait été amené alors à attribuer aux deux types de fibres nerveuses des propriétés trophiques différentes. Mais une courbe de même allure a été obtenue avec un diaphragme réinnervé par le tronc vagal inférieur, qui ne contenait probablement pas de fibres " œsophagiennes "; par conséquent cette interprétation ne semble pas devoir être retenue. Une hypertrophie des fibres musculaires est également observée en pathologie humaine dans des cas de myopathie (WOHLFART, I949), et de poliomyélite. Elle est attribuée à la dénervation partielle du muscle. Le mécanisme ne paraît pas établi; mais c'est sans doute le même qui a agi dans le cas que nous considérons.

\section{2. -- DONNÉES ÉLECTROPHYSIOLOGIQUES}

A. - Avons-nous bien enregistré l'activité de fibres musculaires du diaphragme?

Lorsqu'il a été mis "sous la peau " le diaphragme est en rapport, par sa face externe avec le muscle peaucier, par sa face interne avec le réseau. Nous pouvons donc nous demander si les potentiels que nous avons recueillis proviennent du réseau, du peaucier ou du diaphragme.

a) Le réseau.

Il est effectivement facile de piquer l'aiguille de BRONK dans le réseau, particulièrement si elle a un biseau long. Le diagnostic est simple : étant donné que le réseau, recouvert du péritoine viscéral, glisse à chaque contraction sur le diaphragme, recouvert du péritoine pariétal, 1'aiguille se trouve mobilisée, et son talon subit des mouvements importants qui n'échappent pas à un observateur averti. De plus on recueille à ce moment d'importants artéfacts de mouvements, qui comportent des potentiels lents. Nous avons donc éliminé sans doute possible cette cause d'erreur. 
Quant à la possibilité qu'une aiguille piquée dans le diaphragme recueille à distance des potentiels électriques produits par la contraction du réseau, elle ne peut pas être soutenue. On pourrait présenter de nombreux arguments : haut pouvoir de discrimination des aiguilles utilisées, forme des potentiels, etc. Mais le meilleur nous semble être l'impossibilité dans laquelle nous nous sommes trouvé de recueillir des potentiels lorsque le diaphragme n'était pas réinnervé, alors que la proximité du réseau était la même.

\section{b) Le peaucier.}

Le peaucier est très développé chez les animaux. Nous avons hésité à l'enlever de crainte d'altérer dangereusement l'irrigation de la peau à laquelle il adhère étroitement. Il peut arriver accidentellement que l'aiguille soit piquée dedans.

A première vue ceci ne devrait pas être une cause d'erreur. Il ne devrait pas y avoir de rapport chronologique possible entre la contraction gastrique et celle du peaucier. Dans nos premières expériences, cependant, il semble que cela se soit produit. En particulier, nous avons recueilli au cours d'un enregistrement, des potentiels que nous croyons maintenant être ceux du peaucier, mais qui présentaient les caractéristiques suivantes : on obtenait une salve au moment de chacune des deux pointes de la contraction biphasique du réseau lorsque le ballon enregistreur était très gonflé ; une salve seulement pour la deuxième phase de la contraction (la plus puissante) si le ballon était un peu moins gonflé ; la réponse électrique disparaissait si la distension du réseau était encore diminuée. Nous supposons qu'il s'agissait du réflexe viscéropanniculaire décrit par AshKenaz et SPIEGEL (1935, I937) chez le Chat (contraction réflexe du peaucier en réponse à une excitation douloureuse portée sur un organe abdominal, ou sur le nerf splanchnique). Le ballon enregistreur devait rendre la contraction du réseau douloureuse lorsqu'il était trop gonflé.

Quoi qu'il en soit, nous sommes maintenant attentif à cette cause d'erreur possible. Il est facile de la détecter car, d'une part les potentiels du peaucier sont plus brefs et ont une fréquence plus élevée que ceux du diaphragme réinnervé, d'autre part ils peuvent être déclenchés facilement par la piqûre ou l'excitation tactile de la peau.

\section{B. - S'agit-il de potentiels d'unités motrices réinnervées?}

Il est donc établi que nous avons recueilli l'activité des fibres musculaires diaphragmatiques. Mais il est possible de détecter une activité électrique dans des muscles dénervés : ce sont les potentiels de fibrillation qui peuvent être déclenchés en particulier par l'ébranlement mécanique du muscle. Est-il possible que nous ayons enregistré ce type de potentiels? Nous pouvons répondre par la négative. En effet:

- si certains muscles dénervés dégénèrent très lentement et peuvent donner des potentiels de fibrillation pendant plusieurs dizaines d'années, d'après nos contrôles le diaphragme du Mouton, au contraire, dégénère très vite ;

- les potentiels de fibrillation correspondent à la contraction de fibres musculaires isolées : ils sont brefs et de forme simple (bi- ou triphasiques). Au contraire, nos potentiels étaient longs et le plus souvent polyphasiques, ce qui est typique des unités motrices de réinnervation; 
- enfin si les potentiels avaient été déclenchés par l'ébranlement mécanique de la contraction gastrique, ils l'auraient été tout aussi bien par l'ébranlement du rythme respiratoire.

Nous avons donc bien enregistré l'activité d'unités motrices reconstituées par les fibres vagales.

\section{C. - Pourquoi les potentiels sont-ils polyphasiques et de forme fluctuante?}

A. - Pour les muscles réinnervés, même par leur nerf d'origine (après une section suivie de suture), il est courant que l'électromyogramme soit composé de potentiels polyphasiques. Il n'est donc pas surprenant que tel ait été également le cas pour le diaphragme de nos animaux. Les potentiels faiblement polyphasiques que l'on trouve parfois dans des muscles normaux, peuvent s'expliquer simplement par les conditions de dérivation d'un potentiel propagé dans un milieu conducteur (BISHOP, I937). Mais cette interprétation ne vaut pas pour les " potentiels de réinnervation " très polyphasiques. Pour les expliquer Denslow et HASSETT (I943) font jouer un rôle à la différence de longueur et de diamètre des branches nerveuses auxquelles sont appendus les différents faisceaux de fibres musculaires constitutifs de l'unité motrice : l'organisation de celle-ci n'est certainement pas aussi rigoureuse après une réinnervation. WEDDELL et al. (I944), JASPER et BALLEM (I949) trouvent difficile d'expliquer ainsi des différences de latence de Io à I 5 millisecondes entre la première et la dernière composante de la réponse; ils pensent qu'il faut peut-être faire jouer un rôle à une différence du délai de plaque motrice.

Une différence de latence de 30 millisecondes, pour deux faisceaux musculaires appartenant à la même unité motrice, peut s'expliquer par exemple si les branches nerveuses respectives ont la même longueur, mais une vitesse de conduction égale à 0,6 mètre par seconde pour l'une et 2 mètres par seconde pour l'autre sur une longueur de 26 millimètres ; elle peut également s'expliquer si les fibres ont une vitesse de conduction de 0,6 mètre par seconde, et si l'une est plus longue que l'autre de I $8 \mathrm{~mm}$. Or l'examen histologique des diaphragmes réinnervés montre que la fibre terminale est généralement amyélinique. Dans ces conditions, il ne semble pas indispensable de faire intervenir une différence du délai de plaque motrice pour expliquer la dispersion de la réponse.

B. - Quant aux fuctuations de la forme des potentiels polyphasiques, il faut noter qu'elles ont déjà été observées dans des électromyogrammes de myopathies par KUGELBERG (I949). Il les a attribuées à des perturbations de la propagation des influx. Nous pensons que les fluctuations peuvent s'expliquer, soit par une déficience de la conduction neuro-musculaire, soit par un arrêt de la propagation de l'influx au niveau de l'embranchement des fibres nerveuses. Dans la première hypothèse, un nombre variable de fibres musculaires peut ne pas répondre et l'on doit obtenir une variation graduée de l'intensité de chaque spike. Dans la seconde hypothèse, toutes les fibres musculaires appendues à un même rameau nerveux doivent rester silencieuses ; on doit observer une réponse par tout ou rien des spikes correspondant à une seule sous-unité et une variation discontinue de l'intensité de celles qui traduisent l'activité simultanée de plusieurs sous-unités. D'après l'examen attentif des 
enregistrements, nous ne pouvons pas exclure l'intervention du premier mécanisme, mais nous avons l'impression que la plupart des fluctuations sont dues au second.

Un phénomène semblable avait déjà été envisagé par Eccles et MaLcor.M (I946) : pour expliquer certains faits constatés au niveau de la moelle épinière, ils avaient admis qu'un potentiel se propageant le long d'une fibre afférente n'envahit pas forcément à chaque fois l'ensemble des branches. Pour eux le phénomène pouvait être dû̀ à l'augmentation rapide de la surface de la membrane aux endroits de branchement, ce qui peut diminuer considérablement le coefficient de sécurité de la propagation. Le mécanisme envisagé serait donc identique à celui qui s'oppose souvent à l'envahissement des motoneurones par la voie antidromique. KRNJEvic et Muledi (I958, I959) ont également observé un déficit de la propagation nerveuse dans les préparations phrénique-diaphragme de Rat. Ils admettent qu'il peut se produire surtout aux points de branchement, oì le facteur de sécurité de la transmission est réduit.

\section{3. - Discussion gÉNÉRALE}

Nous avons donc établi les faits suivants :

- les fibres vagales ont envahi le phrénique et atteint le diaphragme,

- elles ont réalisé des plaques motrices sur le diaphragme,

- ces plaques motrices sont fonctionnelles,

-. 1'activité des fibres diaphragmatiques réinnervées est rythmée sur celle de 1'estomac.

Ces divers critères nous permettent d'affirmer que nous avons bien obtenu la réinnervation de fibres musculaires striées par des fibres vagales normalement destinées à l'estomac. Or, il est classiquement admis, chez les Monogastriques, que ce sont des fibres parasympathiques préganglionnaires qui vont constituer des synapses dans la paroi gastrique au niveau des cellules de DoGIEL. De nombreux faits montrent qu'il en est de même chez les Ruminants. En effet, la musculature gastrique de ces animaux est constituée par des fibres musculaires lisses (nous avons vérifié cette donnée classique). Les recherches de KoLossow (I932) et de HABEI. (I956) montrent que les estomacs des Ruminants contiennent un plexus nerveux riche en cellules de DOGIEI, de type I sur lesquelles des fibres nerveuses se terminent par des petits boutons ou des anneaux; des fibres dégénérées peuvent être trouvées autour de ces cellules après section du pneumogastrique thoracique. De plus, il est connu depuis longtemps que l'atropine bloque l'action du vague sur les estomacs des Ruminants ; Brunaun et NAVARro (I954) ont montré qu'il en était de même des ganglioplégiques (nicotine, pentaméthonium, hexaméthonium). Nous avons nous-même vérifié, chez un animal porteur d'une fistule, que l'injection intraveineuse de Ioo $\mathrm{mg}$ d'hexaméthonium (environ $2,5 \mathrm{mg}$ par $\mathrm{kg}$ ) produit un blocage total des contractions du réseau qui dure ro minutes; les contractions reprennent ensuite avec une amplitude progressivement croissante.

Nous avons dit dans l'introduction que la réinnervation d'un muscle strié par des fibres préganglionnaires orthosympathiques a été obtenue à diverses reprises, tandis que la réinnervation par des fibres préganglionnaires parasympathiques n'avait pas fait l'objet d'une étude systématique. La réinnervation du diaphragme par des fibres préganglionnaires vagales a déjà dî cependant être réalisée une fois. 
En effet, dans une courte note préliminaire, BEATTIE (I932) dit que la stimulation du tuber cinereum produit une contraction du diaphragme lorsque le vague a été sectionné après l'émergence des récurrents et suturé avec le phrénique r8o jours auparavant. L'auteur ayant également montré que la stimulation du tuber cinereum produit une augmentation du péristaltisme de l'estomac, effet qui disparaît après la bivagotomie, on peut admettre que la contraction diaphragmatique obtenue était due à une réinnervation du muscle par les fibres préganglionnaires vagales. L'espèce animale utilisée pour ces expériences n'est pas précisée, mais c'était probablement le Chat comme dans les expériences de BEATTIF et al. (I932). Én dépit de l'imprécision du travail de BEATTIE, qui en particulier n'a pas fait de contrôles histologiques, on peut donc conclure que la possibilité pour les fibres préganglionnaires vagales de réinnerver un muscle strié n'est pas propre aux Ruminants, mais existe probablement aussi chez les Monogastriques.

L'aptitude que manifeste ainsi la fibre préganglionnaire parasympathique à constituer une plaque motrice sur une fibre musculaire striée est déjà intéressante en soi. Mais de plus, nous avons vu que cette préparation nous avait permis d'enregistrer chez l'animal éveillé l'activité des unités motrices diaphragmatiques, ce qui revient, à étudier en condition chronique l'activité unitaire des fibres parasympathiques. Nous pensons que cette technique pourrait être appliquée à l'étude de nombreux problèmes.

Reçu pour publication en juillet 1963 .

\section{SUMMARY}

REINNERVATION OF A STRIATED MUSCLE BY PARASYMPATHETIC PREGANGLIONIC FIBRES. APYLICATION TO THE RECORD OF THE ACTIVITY OF THE EFFERENT VAGAL FIBRES IN AWAKE ANIMALS.

By means of a cross union of the vagus and phrenic nerves behind the emergence spot of the recurrent nerve, the diaphragm has been reinnerved by vagal motor fibres in 6 sheep and 6 goats. A window in the thoracic wall changed the diaphragm into a subcutaneous muscle, so that its electromyographic activity might be easily collected in the awake animal via BRONK needles. The records thus obtained and histological controls made after killing the animals showed that :

I) the vagal fibres have grown into the phrenic and reached the diaphragm,

2) they have constitued motor end plates on this muscle,

3) these motor end plates are functional,

4) the activity of the diaphragmatic muscle fibres reinnerved by the left vagus is rhythmed on this of the stomach provoked by the intact right vagus.

This demonstrates that the vagal preganglionic fibres which normally supply the stomach, can constitute a functional neuromuscular junction on striated muscular fibres.

The electrical activity of the reinnerved motor units can be easily collected in the awake animal via BRoNk needles. So the cross union of heterogeneous nerves can be a valuable technique for studying the unitary activity of the efferent vagal fibres in chronic animals.

\section{RÉFÉRENCES BIBLIOGRAPHIQUES}

ANOKHIN P., Iwanow A., I936. Experimentelle Veränderung der phylogenetischen Verbindungen im Gebiet des Nervus vagus. Pflüger's Arch. ges. Physiol., 237, 536-557.

Ashkenaz D. M., 1937. An experimental analysis of centripetal visceral pathways based upon the visceropannicular reflex. Amer. J. Physiol., 120, 587-596.

Beattie J., I932. The relation of the tuber cinereum to gastric and cardiac functions. Canad. med. Ass. $J ., 26,278$. 
Beattie J., Duel A.B., Ballance C., I932. The effects of stimulation of the hypothalamic pupillodilator centre after successful anastomoses between the cervical sympathetic and certain motor nerves. $J$. Anat. Lond., 66, 283-299.

BerRy C. M., Hinsey J.C., I946. The recovery of diameter and impulse conduction in regenerating nerve fibers. Ann. N. Y. Acad. Sci., 47, 559-574.

Bishop G. H., I937. La théorie des circuits locaux permet-elle de prévoir la forme du potentiel d'action? Arch. int. Physiol., 45, 273-297.

Brunaud M., Navarro J., I954. Modifications, sous l'influence de substances ganglioplégiques, de l'action du nerf vague sur les estomacs du Mouton. J. Physiol. (Fr.), 46, 272-276.

Calugareanu D., Henri V., igoo. Expériences sur la structure croisée des nerfs de différentes sortes. Nerf lingual avec le nerf hypoglosse, nerf hypoglosse avec le nerf pneumogastrique. $C$. R. Soc. Biol., Paris, $52,5 \circ 3$.

Denslow J.S., HassetT C. C., I943. The polyphasic action currents of the motor unit complex. Amer. J. Physiol., 139, 652-66o.

Dumont B. L., Paris J., 1959. Application des méthodes de coloration différentielle du tissu musculaire et du tissu conjonctif aux travaux de recherches sur la viande. Communication $5^{\mathrm{e}}$ Réunion Inst. Rech. sur les viandes, Paris.

DUSSARDIER M., 1960. Recherches sur le contrôle bulbaire de la motrocité gasirique chez les Ruminants. Thèse Doctorat ès-Siences, Paris. INRA édit.

Dussardier M., ig6i a. Technique de fistulation de la région antérieure du rumen par la voie thoracique chez les petits Ruminants. Ann. Biol. anim. Bioch. Biophys. 1, I I 3-i I6.

DUSSARdier M., 196r $b$. Effets de la vagotomie intrathoracique partielle sur la survie et la croissance du Mouton. Ann. Biol. anim. Bioch. Biophys., 1, I4I-I 44 .

Dussardier M., 1962. Répartition des fibres nerveuses sensitives et motrices dans le tronc vagal thoracique du Mouton. Ann. Biol. anim. Bioch. Biophys., 2, 3+5-36o.

Eccles J. C., Malcolm J. L., 1946. Dorsal root potentials of the spinal cord. J. Neurophysiol., 9, I39160.

Guti L., FRANK K., I959. Restoration of diaphragmatic function following vagophrenic anastomosis in the rat. Exper. Neurol., 1, I-12.

IABEL R. E., I956. A study of the innervation of the ruminant stomach. Cornell vet., 46, 555-628.

Hammond W.S., Hinsey J. C., 1945. The diameters of the nerve fibers in nomal and regenerating nerves. J. comp. Veurol., 83, 79-80.

Hillarp N. A,, 1946. Structure of the synapse and the peripheral innervation apparatus of the automatic nervous system. Acta anat., supplément IV, I-I 53.

JASPER II., BALLEM G., I949. Unipolar electronyogranıs of normal and denervated human muscle. J. Neurophysiol., 12, $23^{1-244}$.

Kolossow N. G., r932. Observations concernant l'innervation de la voie digestive chez les Ruminants. Trav. Lab. Invest. biol. Univ. Madr., 28, 345-368.

KRnJevic K., Miledi R., 1958. Failure of neuromuscular propagation in rats. J. Phy'siol. (G. B.), 140, 440-461.

KrnjeVic K., Miledi R., 1959. Presynaptic failure of neuromuscular propagation in rats. J. Physiol. (G. B.), 149, I-22.

Kugelberg E., I949. Electromyography in muscular dystrophies. Differentiation between dystrophies and chronic lower motor neurone lesions. J. Neurol. Psychiatr., 12, 129.

LANGLey J. N., ANDERSON H. K., I904. On the union of the fifth cervical nerve with the superior cervical ganglion. J. Physiol. (G. B.), 30, 439-442.

Langley J. N., Anderson H. K., 1904. The union of different kinds of nerve fibres. J. Physiol. (G. B.), $31,365-39 \mathrm{I}$.

Mislavsky M., I902. Suture du sympathique cervical et du récurrent et centres corticaux du larynx. C.R. Soc. Biol., Paris, 54, 84 I.

Philipeaux J. M., Vulpian A., 1863. Recherches expérimentales sur la réunion bout à bout de nerfs de fonctions différentes. J. de la Physiol. (Fr.), 6, 42I-455.

Reichert E. T., 1885. Observations on the regeneration of the vagus and hypoglossal nerves. Amer. J. med. Sci., 89, $146-\mathrm{I} 59$.

Weddell G., Feinstein B., Pattle R. E., i 944 . The electrical activity of voluntary muscle in man under normal and pathological conditions. Brain, 67, $17^{8-257}$.

Wohlfart G., I949. Muscular atrophy in diseases of the lower motor neuron. Arch. Neurol. Psychialr., Lond., 61, 599-620. 